\title{
THE MAXIMAL OPERATOR SPACE OF A NORMED SPACE
}

\author{
by VERN I. PAULSEN*
}

(Received 27th June 1994)

\begin{abstract}
We obtain some new results about the maximal operator space structure which can be put on a normed space. These results are used to prove some dilation results for contractive linear maps from a normed space into $B(H)$. Finally, we prove $C B(\operatorname{MIN}(X), \operatorname{MAX}(Y))=\Gamma_{2}^{*}(X, Y)$ and apply this result to prove some new Grothendieck-type inequalities and some new estimates on spans of "free" unitaries.
\end{abstract}

1991 Mathematics subject classification: 46L05, 46M05.

\section{Introduction}

Given any (complex) vector space $X$, let $M_{n}(X)$ denote the vector space of $n \times n$ matrices with entries from $X$. If $B(H)$ denotes the bounded linear operators on a Hilbert space $H$ then $M_{n}(B(H))$ is endowed with a natural norm via the identification, $M_{n}(B(H))=B(H \oplus \cdots \oplus H)$ ( $n$ copies). If $X$ is any subspace of $B(H)$ then the inclusion $M_{n}(X) \subseteq M_{n}(B(H))$ endows $M_{n}(X)$ with a norm. These are generally called a matrixnorm on $X$. Subspaces of $B(H)$ are called operator spaces to indicate that they are more than normed spaces, but normed spaces with a distinguished family of norms on the spaces, $M_{n}(X)$.

If $X$ is only a normed space, then any linear isometry $\phi: X \rightarrow B(H)$ for some $H$ endows each $M_{n}(X)$ with a norm, i.e. makes $X$ an operator space. Generally, for a normed space $X$ the norms on $M_{n}(X)$ thus obtained are not unique. In [3] it was noted that among all such matrix norms there is a minimal and maximal family. These are operator space structures on $X$ which satisfy

$$
\left\|\left(x_{i j}\right)\right\|_{\mathrm{MIN}} \leqq\left\|\left(\phi\left(x_{i j}\right)\right)\right\| \leqq\left\|\left(x_{i j}\right)\right\|_{\mathrm{MAX}}
$$

for any isometry $\phi: X \rightarrow B(H)$ for all $n$ and for all $\left(x_{i j}\right)$ in $M_{n}(X)$. We write $\operatorname{MIN}(X)$ and $\operatorname{MAX}(X)$ to denote $X$ endowed with these matrix-norms.

In [10] we introduced the $\alpha$ constant of a normed space $X$, defined by

$$
\alpha(X)=\sup \left\{\left\|\left(x_{i j}\right)\right\|_{\text {MAX }}:\left\|\left(x_{i j}\right)\right\|_{\text {MIN }}=1\right\}
$$

where the supremum is over all $n$ and $\left(x_{i j}\right)$ in $M_{n}(X)$. Thus, $\alpha(X) \geqq 1$ always and $\alpha(X)=1$

*Research supported in part by a grant from the NSF. 
precisely when every isometric embedding of $X$ into $B(H)$ induces the same matrix norm structure on $X$. In [10] we also proved that $\alpha(X)>1$ for any normed space $X$ of dimension greater than or equal to 5 and that $\alpha(X)$ is infinite whenever $X$ is infinite dimensional.

Currently, there is a rapidly evolving local theory of finite dimensional operator spaces which has many parallels with the local theory of Banach spaces. It is clear that the operator spaces $\operatorname{MIN}(X)$ and $\operatorname{MAX}(X)$ together with the measure $\alpha(X)$ of how different these spaces can be plays a role in this theory.

In this paper we give a new formula for the matrix-norm on $\operatorname{MAX}(X)$. Originally this matrix-norm was defined extrinsically, by taking a supremum over all isometric representations of $X$ as a space of operators. Our new formula has the advantage of being intrinsic, depending only on $X$ and it is expressed as an infimum, so that interplay between the two definitions allows for better estimates. This result also allows for a dilation theoretic characterization of the MAX norm.

We then turn our attention to $\operatorname{MAX}\left(\ell_{1}^{n}\right)$ where $\ell_{1}^{n}$ denotes the $n$-dimensional $\ell_{1}$-space. This operator space is completely isometrically isomorphic to the span of $1, u_{1}, \ldots, u_{n-1}$ in the universal $C^{*}$-algebra of the free group on $n-1$ generators, $C^{*}\left(\mathbb{F}_{n-1}\right)$ where, $u_{1}, \ldots, u_{n-1}$ denote the canonical generators of $\mathbb{F}_{n-1}$. Using this representation we are able to prove via dilation techniques that $\alpha\left(\ell_{1}^{n}\right) \leqq \sqrt{n-1}$. While this estimate is only a slight improvement on our estimates in [10] we believe that it is significant because we have been unable to obtain this sharper estimate via Banach space methods. In [13] a "smallest" $C^{*}$-algebra generated by an operator space was introduced, in analogy with Arveson and Hamana's boundary $C^{*}$-algebra of an operator system. For $\operatorname{MAX}\left(\ell_{1}^{n}\right)$ this algebra is $C^{*}\left(\mathbb{F}_{n-1}\right)$, and for this reason we believe that the above estimate might be sharp.

Finally, for any Banach spaces $X$ and $Y$ we are able to prove that $C B(\operatorname{MIN}(X)$, $\operatorname{MAX}(Y))$ is equal to the space $\Gamma_{2}^{*}(X, Y)$ of maps $T$ from $X$ to $Y$ which have a factorization through Hilbert space $T=A B, B: X \rightarrow H, A: H \rightarrow Y$ such that the 2-summing norms, $\pi_{2}(B)$ and $\pi_{2}\left(A^{*}\right)$ are both finite. In fact we prove that $\frac{1}{k} \gamma_{2}^{*}(T) \leqq\|T\|_{c b} \leqq \gamma_{2}^{*}(T)$ where $k$ is a universal constant, $\sqrt{2} \leqq k \leqq 2$ and $\gamma_{2}^{*}(T)=\inf \left\{\pi_{2}(B) \pi_{2}\left(A^{*}\right)\right\}$ where the infimum is taken over all factorizations through Hilbert space. This result was obtained with Pisier and has also been obtained independently by $M$. Junge with a larger constant, who together with Pisier [7] have applied it to obtain their proof that the minimal and maximal $C^{*}$-tensor norms on $B(H) \otimes B(H)$ are different. The main advantage of the proof that we present here is that it gives a sharp characterization of the value $k$, namely,

$$
k=\lim _{n \rightarrow \infty} n / \alpha\left(\ell_{n}^{n}\right)
$$

and shows the importance of this ratio in the local "metric" theory of operator spaces.

Finally, applying some results from [3] we show that the above result implies some equivalences between tensor norms in the operator space category which are natural generalizations of Grothendieck's result on the equivalance of the Hilbertian and 
projective norms for tensor products of $\ell^{\infty}$-spaces. These give rise to a new estimate on "free" unitaries.

\section{The MAX operator space}

Let $X$ be a normed space and for each $n$ define a norm on $M_{n}(X)$ via,

$$
\left\|\left(x_{i j}\right)\right\|_{\text {MAX }}=\sup \left\{\left\|\left(\phi\left(X_{i j}\right)\right)\right\|: \phi: X \rightarrow B(H),\|\phi\| \leqq 1\right\} .
$$

We let $\operatorname{MAX}(X)$ denote $X$ together with this family of matrix-norms. It is easily seen (by considering the direct sum of a sufficiently large family of such $\phi$ 's) that $\operatorname{MAX}(X)$ can be embedded completely isometrically as a subspace of $B(H)$ for some $H$. This also follows from Ruan's theorem [12]. Clearly the MAX norms dominate any other matrixnorm on $X$ which has the property that they can be realized completely isometrically as a subspace of some $B(H)$, i.e., which satisfy Ruan's axioms [12]. Similarly, if we set

$$
\left\|\left(x_{i j}\right)\right\|_{\text {MIN }}=\sup \left\{\left\|\left(f\left(x_{i j}\right)\right)\right\|: f \in X^{*},\|f\| \leqq 1\right\}
$$

and let $\operatorname{MIN}(X)$ denote $X$ together with this family of matrix-norms, then $\operatorname{MIN}(X)$ can be represented completely isometrically as a space of operators (in fact $\operatorname{MIN}(X) \subseteq C\left(X_{1}^{*}\right)$ completely isometrically) and every other family of matrix norms with the property that they can be realized completely isometrically as a subspace of some $B(H)$ dominate these norms. See [10] for more details.

The above definition of the MAX norms are extrinsic and our first theorem gives an intrinsic characterization of these norms. We remark that it is well-known and easily checked that,

$$
\left.\left\|\left(x_{i j}\right)\right\|_{\mathrm{MIN}}=\sup \left\|\sum \lambda_{i} u_{j} x_{i j}\right\|: \sum\left|\lambda_{i}\right|^{2} \leqq 1, \sum\left|u_{i}\right|^{2} \leqq 1\right\}
$$

Theorem 2.1. Let $X$ be a normed space. Then for any $n$ and any $\left(x_{i j}\right)$ in $M_{n}(X)$, $\left\|\left(x_{i j}\right)\right\|_{\operatorname{MAX}}=\inf \{\|A\|\|B\|\}$ where the infimum is taken over all pairs of scalar matrices $A=\left(a_{i j}\right), B=\left(b_{i j}\right)$ of sizes $n \times k$ and $k \times n$ for which there exist $y_{1}, \ldots, y_{k}$ in $X$ satisfying, $\left\|y_{i}\right\| \leqq 1,1 \leqq i \leqq k$ and $x_{i j}=\sum_{\ell=1}^{k} a_{i \ell} y_{\ell} b_{\ell j}$.

Proof. Let $\left\|\left(x_{i j}\right) \mid\right\|$ denote this infimum. If $\phi: X \rightarrow B(H)$ is any contractive linear map $A, B,\left\{y_{1}, \ldots, y_{k}\right\}$ are as above then,

$$
\left(\phi\left(x_{i j}\right)\right)=A\left(\begin{array}{lll}
\phi\left(y_{1}\right) & & 0 \\
& \ddots & \\
0 & & \phi\left(y_{k}\right)
\end{array}\right) B
$$

and hence $\left\|\left(x_{i j}\right)\right\|_{\text {MAX }} \leqq\left\|\left|\left(x_{i j}\right)\right|\right\|$. 
To complete the proof of the theorem it will be enough to show that $\mathbf{X}$ together with the matrix norms $\||\cdot|\|$ satisfy Ruan's axioms for an operator space [12].

Clearly, if $C, D$ are matrices of scalars then $\left\|\left|C\left(x_{i j}\right) D\right|\right\| \leqq\|C\|\left\|\left|\left(x_{i j}\right)\right|\right\| D \|$. Thus, it remains to show that if $X=\left(x_{i j}\right)$ is in $M_{n}(\mathbf{X})$ and $X^{\prime}=\left(x_{i j}^{\prime}\right)$ is in $M_{m}(\mathbf{X})$, then

$$
\left\|\left(\begin{array}{cc}
X & 0 \\
0 & X^{\prime}
\end{array}\right)\right\| \leqq \max \left\{\||X|\|,\left\|\left|X^{\prime}\right|\right\|\right\},
$$

Write $X=A Y B$ and $X^{\prime}=A^{\prime} Y^{\prime} B^{\prime}$ where $A, B, A^{\prime}$ and $B^{\prime}$ are matrices of scalars and $Y$ and $Y^{\prime}$ are diagonal matrices with entries from $X$ which are all of norm less than 1 . By scaling we may assume that we have chosen such a representation satisfying $\|A\|=$ $\|B\| \leqq\||X|\|^{1 / 2}+\varepsilon,\left\|A^{\prime}\right\|=\left\|B^{\prime}\right\| \leqq\left\|\left|X^{\prime}\right|\right\|^{1 / 2}+\varepsilon$ for any $\varepsilon>0$. Hence

$$
\left(\begin{array}{cc}
X & 0 \\
0 & X^{\prime}
\end{array}\right)=\left(\begin{array}{cc}
A & 0 \\
0 & A^{\prime}
\end{array}\right)\left(\begin{array}{cc}
Y & 0 \\
0 & Y^{\prime}
\end{array}\right)\left(\begin{array}{cc}
B & 0 \\
0 & B^{\prime}
\end{array}\right)
$$

from which it follows that

$$
\left\|\left|\left(\begin{array}{cc}
X & 0 \\
0 & X^{\prime}
\end{array}\right)\right|\right\| \leqq \max \left\{\||X|\|,\left\|\left|X^{\prime}\right|\right\|\right\}+\varepsilon
$$

Note: In matrix notation we have that

$$
\left(x_{i j}\right)=A\left(\begin{array}{lll}
y_{1} & & \\
& \ddots & \\
& & y_{n}
\end{array}\right) B .
$$

If $\mathbf{X}$ has a basis $\left\{e_{1}, \ldots, e_{n}\right\}$ and $\left(x_{i j}\right)$ is in $M_{m}(\mathbf{X})$ with $x_{i j}=\sum_{k=1}^{n} a_{i j}^{k} e_{k}$ then we write

$$
\left(x_{i j}\right)=\sum_{k=1}^{m} A_{k} \otimes e_{k} \quad \text { where } A_{k}=\left(a_{i j}^{k}\right) .
$$

Let $G=\left\{\left(\lambda_{1}, \ldots, \lambda_{n}\right) \in \mathbb{C}^{n}:\left\|\sum \lambda_{i} e_{i}\right\| \leqq 1\right\}$ and let $G^{*}=\left\{\left(u_{1}, \ldots, u_{n}\right) \in \mathbb{C}^{n}: \sum \lambda_{i} u_{i} \leqq 1, \forall \lambda \in G\right\}$ denote the polar. We write $X=\left(\mathbb{C}^{n}, G\right)$ and $X^{*}=\left(\mathbb{C}^{n}, G^{*}\right)$. Clearly $X^{*}$ is the dual of $X$ with the dot product pairing.

Proposition 2.2. Let $X$ be a finite dimensional normed space with basis $\left\{e_{1}, \ldots, e_{n}\right\}$ and let $G$ be as above, i.e. $X=\left(\mathbb{C}^{n}, G\right)$. The following are equivalent for any matrix $\left(x_{i j}\right)=\sum_{k=1}^{n} A_{k} \otimes e_{k}$ in $M_{m}(X)$

(a) $\left\|\left(x_{i j}\right)\right\|_{\operatorname{MAX}(X)}<1$.

(b) for some $r$ there exists $r \times r$ diagonal matrices $D_{k}=\operatorname{diag}\left(d_{k 1}, \ldots, d_{k r}\right), k=1, \ldots, n$ 
$m \times r$ matrices $B$ and $C^{*}$ with $\|B\|\|C\|<1$ such that $A_{k}=B D_{k} C$ and $\left(d_{1 j}, \ldots, d_{n j}\right) \in G$ for $j=1, \ldots, r$.

(c) There exists a Hilbert space $H$, commuting normal operators $N_{k}, k=1, \ldots, n$ on $H$ and $B, C^{*}: H \rightarrow \mathbb{C}^{m}$ with $\|B\|\|C\|<1$ such that $A_{k}=B N_{k} C$ and $\sigma\left(N_{1}, \ldots, N_{n}\right) \subseteq G$.

(d) Same as (c) but with $\sigma\left(N_{1}, \ldots, N_{n}\right) \subseteq E(G)$ where $E(G)$ denotes any set whose absolute convex hull is $G$.

(e) $\left\|\sum_{i=1}^{n} A_{i} \otimes B_{i}\right\|<1 \quad$ for any $\left\{B_{1}, \ldots, B_{n}\right\}$ satisfying $\left\|\sum_{i=1}^{n} u_{i} B_{i}\right\| \leqq 1$ for all $\left(u_{1}, \ldots, u_{n}\right) \in G^{*}$.

Proof. To see the equivalence of (a) and (b) let $y_{j}=\sum_{k=1}^{n} d_{k j} e_{k}$ and apply the Theorem 2.1. The equivalence of (a) and (e) was proven in [10].

Clearly (b) implies (c) by setting $N_{k}=D_{k}$. To see that (c) implies (a), let $K$ be a Hilbert space and let $\phi: X \rightarrow B(K)$ be contractive. Set $\phi\left(e_{i}\right)=B_{i}$ so that $\left\|\sum \lambda_{i} B_{i}\right\|<1$ for all $\left(\lambda_{1}, \ldots, \lambda_{n}\right) \in G$. We will show that $\left(\phi\left(x_{i j}\right)\right)=\sum A_{k} \otimes B_{k}$ has norm less than one, since $\phi$ was arbitrary this will prove that $\left\|\left(x_{i j}\right)\right\|_{\text {MAx }} \leqq 1$.

To this end note that $\sum A_{k} \otimes B_{k}=B \otimes 1\left(\sum N_{k} \otimes B_{k}\right) C \otimes 1$ and from $C^{*}$-algebra theory,

$$
\left\|\sum_{k=1}^{n} N_{k} \otimes B_{k}\right\|=\sup \left\{\left\|\lambda_{k} B_{k}\right\|:\left(\lambda_{1}, \ldots, \lambda_{n}\right) \in \sigma\left(N_{1}, \ldots, N_{n}\right)\right\} \leqq 1
$$

Clearly, (d) implies (c). Finally to see (c) implies (d), consider span $\left\{z_{1}, \ldots, z_{n}\right\} \subseteq C(G)$ and $M=\operatorname{span}\left\{z_{1}, \ldots, z_{n}\right) \subseteq C(E(G))$. Since these spaces are completely isometrically isomorphic any commuting normals $\left(N_{1}, \ldots, N_{n}\right)$ with $\sigma\left(N_{1}, \ldots, N_{n}\right) \subseteq G$ give rise to a completely contractive map $\phi$ on $M$ by setting $\phi\left(z_{i}\right)=N_{i}$. Applying the generalized Stinespring dilation theorem [9, Theorem 7.4] to this map allows us to write $N_{i}=X \pi\left(z_{i}\right) Y$ where $\pi: C(E(G)) \rightarrow B(K)$ is a *-homomorphism and $\|X\|\|Y\|=1$. Obviously, $\left(\pi\left(z_{1}\right), \ldots, \pi\left(z_{n}\right)\right)$ is a commuting $n$-tuple of normals with spectrum in $E(G)$. Hence any $n$-tuple satisfying (c) can be replaced by one satisfying (d).

Remark. One can also deduce Proposition 2.2 from Blecher's result that $\operatorname{MIN}(X)^{*}=$ $\operatorname{MAX}\left(X^{*}\right)$ completely isometrically [2].

Also, in the case $\left\|\left(x_{i j}\right)\right\|_{\operatorname{MAX}(X)}=1$ in (c) and (d) one has $\|B\|\|C\|=1$ while in (b) the best that can be said is that for every $\varepsilon>0$ one has $B_{\varepsilon}$ and $C_{\varepsilon}$ with $\left\|B_{\varepsilon}\right\|\left\|C_{\varepsilon}\right\|<1+\varepsilon$.

The above result leads to another characterization of the $\alpha$ constant.

Corollary 2.3. Let $X=\left(\mathbb{C}^{n}, G\right)$ be as above. There is a constant $\alpha$ such that for any $k$ if $A_{1}, \ldots, A_{n}$ are any $k \times k$ matrices satisfying $\left\|\sum \lambda_{i} A_{i}\right\|<1$ for all $\left(\lambda_{1}, \ldots, \lambda_{n}\right) \in G$ then there exist diagonal matrices $D_{1}, \ldots, D_{n}$ (of possibly larger size) and matrices $B, C$ satisfying, $A_{i}=B D_{i} C$ for $i=1, \ldots, n,\|B\|\|C\|<\alpha$, and $\sigma\left(D_{1}, \ldots, D_{n}\right) \subseteq G^{*}$. Moreover, the least positive constant satisfying this inequality is $\alpha(X)$.

Proof. The condition, $\left\|\sum \lambda_{i} A_{i}\right\|<1$ for all $\left(\lambda_{1}, \ldots, \lambda_{n}\right) \in G$ is equivalent to requiring 
that $\left\|\sum A_{i} \otimes e_{i}\right\|_{\mathrm{MIN}\left(X^{*}\right)}<1$. Hence, $\left\|\sum A_{i} \otimes e_{i}\right\|_{\mathrm{MAX}\left(X^{*}\right)}<\alpha\left(X^{*}\right)$ and the $A_{i}$ 's have the desired representation by Proposition 2.2. Moreover, by Proposition 2.2 any $A_{i}$ 's which have such a representation with a fixed constant $\alpha$ satisfy $\left\|\sum A_{i} \otimes e_{i}\right\|_{\operatorname{MIN}\left(X^{*}\right)}<\alpha$. Hence, the least positive $\alpha$ is $\alpha\left(X^{*}\right)=\alpha(X)$ by [10, Proposition 2.4].

Corollary 2.4. The constant $\alpha\left(\ell_{\infty}^{n}\right)$ is the least positive constant $\alpha$ such that if $A_{1}, \ldots, A_{n}$ are any contractions then there will exists $n$ commuting unitaries $U_{1}, \ldots, U_{n}$ and operators $B, C$ satisfying $\|B\|\|C\| \leqq \alpha$, and $A_{k}=B U_{k} C$ for $k=1, \ldots, n$.

Proof. The result follows as in the proof of Corollary 2.3 applying also the remark following Proposition 2.2.

In Section 3 we prove that, $\alpha\left(\ell_{n}^{\infty}\right)=\alpha\left(\ell_{n}^{\prime}\right) \leqq \sqrt{n-1}$.

We note that if $F \subseteq X$ is a subspace and $x_{i j} \in F$ then the norm of $\left\|\left(x_{i j}\right)\right\|$ in $M_{n}(\operatorname{MAX}(F))$ can be larger than in $M_{n}(\operatorname{MAX}(X))$. However Theorem 2.1 implies that the MAX operator space structure is "local" in the following sense.

Corollary 2.5. Let $x_{i j} \in X, i, j=1, \ldots, n$ then $\left\|\left(x_{i j}\right)\right\|_{M_{n}(\operatorname{MAX}(X))}=\inf \left\{\left\|\left(x_{i j}\right)\right\|_{M_{n}(\operatorname{MAX}(F))}\right.$ : $x_{i j} \in F$ and $F \subseteq X$, finite dimensional $\}$.

\section{A representation of $\operatorname{MAX}\left(\ell_{n}^{1}\right)$}

Let $\mathbb{F}_{n}$ denote the free group on $n$ generators $g_{1}, \ldots, g_{n}$, let $C^{*}\left(\mathbb{F}_{n}\right)$ denote the full group $C^{*}$-algebra of $\mathbb{F}_{n}$ and let $U_{1}, \ldots, U_{n}$ denote the unitaries in $C^{*}\left(\mathbb{F}_{n}\right)$ that are the images of $g_{1}, \ldots, g_{n}$. Recall that $C^{*}\left(\mathbb{F}_{n}\right)$ is characterized by the universal property that if $V_{1}, \ldots, V_{n}$ are any unitaries on a Hilbert space $H$, then there exists a $*$-homomorphism $\pi: C^{*}\left(\mathbb{F}_{n}\right) \rightarrow B(H)$ with $\pi\left(U_{i}\right)=V_{i}, i=1, \ldots, n$. It is known (see for example [13]) that $\operatorname{MAX}\left(\ell_{n}^{1}\right)$ is completely isometrically isomorphic to $\operatorname{span}\left\{U_{1}, \ldots, U_{n}\right\} \subseteq C^{*}\left(\mathbb{F}_{n}\right)$ via the map which sends the canonical basis vectors $e_{i}$ to $U_{i}, i=1, \ldots, n$. To see this one notes that $\phi: \ell_{n}^{1} \rightarrow B(H)$ is contractive if and only if $\left\|\phi\left(e_{i}\right)\right\| \leqq 1$ and then takes the noncommuting unitary dilation of the $\left\{\phi\left(e_{i}\right)\right\}$. For this reason further knowledge about $\operatorname{MAX}\left(\ell_{m}^{1}\right)$ is of some independent interest because it yields knowledge about free unitaries. We gather these results in this section.

Theorem 3.1. Let $U_{1}, \ldots, U_{n}$ be generators of $C^{*}\left(\mathbb{F}_{n}\right)$ and let $A_{1}, \ldots, A_{n}$ be in $M_{m}$. Then $\left\|\sum_{k=1}^{n} A_{k} \otimes U_{k}\right\|<1$ if and only if for some $\ell$ there exist $m \times \ell$ scalar matrices $C_{1}, \ldots, C_{n}$ and $\ell \times m$ scalar matrices $D_{1}, \ldots, D_{n}$ satisfying $A_{k}=C_{k} D_{k}, k=1, \ldots, n$ and

$$
\left\|\sum C_{k} C_{k}^{*}\right\|\left\|\sum D_{k}^{*} D_{k}\right\|<1 \text {. }
$$

Proof. We have $\left\|\sum A_{k} \otimes e_{k}\right\|_{\operatorname{MAX}\left(\ell_{n}^{1}\right)}=\left\|\sum A_{k} \otimes U_{k}\right\|$. Let $\left(x_{i j}\right)=\sum A_{k} \otimes e_{k}$ be in $M_{m}\left(\ell_{n}^{1}\right)$. By Theorem 2.1 there exist matrices of scalars $S=\left(s_{i \ell}\right)$ and $T=\left(t_{\ell j}\right)$ which are $m \times r$ and $r \times m$, respectively, and vectors $y_{i}$ in $\ell_{n}^{1}$ with $\left\|y_{\ell}\right\| \leqq 1,1 \leqq \ell \leqq r$ such that $\|S\|<1,\|T\|<1$ and $x_{i j}=\sum_{\ell=1}^{r} s_{i \ell} y_{\ell} t_{\ell j}$. Write $y_{\ell}=\sum_{k=1}^{n} \alpha_{\ell, k} e_{k}$ and choose scalars $\beta_{\ell, k}, \gamma_{\ell, k}$ such that 
$\left|\alpha_{\ell, k}\right|^{1 / 2}=\left|\beta_{\ell . k}\right|=\left|\gamma_{\ell . k}\right|$ and $\beta_{\ell, k} \gamma_{\ell, k}=\alpha_{\ell k}$. We then have that $x_{i j}=\sum_{k=1}^{n}\left(\sum_{\ell=1}^{r} s_{i \ell} \beta_{\ell k} \gamma_{\ell k} t_{\ell j}\right) e_{k}$. Let $E_{k}$ be the diagonal matrix whose diagonal entries are $\beta_{1 k}, \ldots, \beta_{r k}$, let $F_{k}$ be the diagonal matrix whose diagonal entries are $\gamma_{1 k}, \ldots, \gamma_{r k}$ and set $C_{k}=S E_{k}, D_{k}=F_{k} T$. We have that $A_{k}=C_{k} D_{k}$ and $\left\|\sum C_{k} C_{k}^{*}\right\|=\left\|\sum S E_{k} E_{k}^{*} S^{*}\right\| \leqq\left\|\sum E_{k} E_{k}^{*}\right\| \leqq 1$ since $\sum E_{k} E_{k}^{*}$ is a diagonal matrix whose $i$-th diagonal entry is $\left\|y_{i}\right\|$. Similarly, $\left\|\sum D_{k}^{*} D_{k}\right\| \leqq 1$.

Remark. We mention an alternate way to prove the above result via the tensor product machinery. It is well-known that $\operatorname{MIN}\left(\ell_{n}^{\infty}\right)$ can be represented completely isometrically as the diagonal matrices in $M_{n}$. Thus, the adjoint of this inclusion gives a complete quotient map $q: M_{n}^{*} \rightarrow \operatorname{MIN}\left(\ell_{n}^{\infty}\right)^{*}=\operatorname{MAX}\left(\ell_{n}^{1}\right)$. Chasing through these identifications one recovers the above result.

Corollary 3.2. If $A_{1}, \ldots, A_{n}$ are matrices such that $\left\|\sum_{i=1}^{n} \lambda_{i} A_{i}\right\|<1$ for all $\left|\lambda_{i}\right| \leqq 1$, then there exist matrices $C_{1}, \ldots, C_{n}$ and $D_{1}, \ldots, D_{n}$ such that $A_{k}=C_{k} D_{k}, k=1, \ldots, n$ and $\left\|\sum C_{k} C_{k}^{*}\right\|\left\|\sum D_{k}^{*} D_{k}\right\|<\alpha\left(\ell_{n}^{1}\right)^{2}$. Moreover, $\alpha\left(\ell_{n}^{1}\right)$ is the least positive constant satisfying this inequality.

Proof. The hypotheses are equivalent to assuming that $\left\|\sum A_{i} \otimes e_{i}\right\|_{\operatorname{MiN}\left(\ell_{n}^{\prime}\right)}<1$. Hence $\left\|\sum A_{i} \otimes e_{i}\right\|_{\operatorname{MAX}\left(\ell_{n}^{1}\right)}<\alpha\left(\ell_{n}^{1}\right)$ and this constant is the least constant satisfying this condition for all $n$. The result follows as in the proof of Corollary 2.3 substituting Theorem 3.1 where Proposition 2.2 applied before.

We now turn our attention to the problem of estimating $\alpha\left(\ell_{n}^{1}\right)$. In [10, Theorem 2.16] it was shown $\sqrt{n / 2} \leqq \alpha\left(\ell_{n}^{1}\right) \leqq \sqrt{n}$. Note that if $A_{1}, \ldots, A_{n}$ are matrices satisfying the hypotheses of Corollary 3.2 and we set $C_{k}=A_{k}, D_{k}=I$ then $A_{k}=C_{k} D_{k}$ and $\left\|\sum D_{k}^{*} D_{k}\right\|=n$ while one can easily see by using the fact that $\left\|\sum e^{i \theta_{k}} A_{k}\right\|<1$ and integrating around the $n$ torus that $\left\|\sum C_{k} C_{k}^{*}\right\|<n$. Thus, applying Corollary 3.2 we have a new proof that $\alpha\left(\ell_{n}^{1}\right) \leqq \sqrt{n}$. We shall now obtain a slight improvement of that estimate by obtaining another characterization of $\alpha\left(\ell_{n}^{1}\right)$.

As we remarked earlier $\operatorname{MAX}\left(\ell_{n}^{1}\right)$ embeds completely isometrically in $C^{*}\left(\mathbb{F}_{n}\right)$ by sending $e_{i}$ to $U_{i}$. This representation is not best in some sense which we will make precise in a moment. Note that multiplication by $U_{1}^{*}$ is a complete isometry and hence sending $e_{i}$ to $U_{1}^{*} U_{i}$ induces a new completely isometric represntation of $\operatorname{MAX}\left(\ell_{n}^{1}\right)$. Also, $I=U_{1}^{*} U_{1}, V_{1}=U_{1}^{*} U_{2}, \ldots, V_{n-1}=U_{1}^{*} U_{n}$ can be easily seen to generate $C^{*}\left(\mathbb{F}_{n-1}\right)$ by using the universal properties. Zhang [14] shows that this representation is minimal in the sense that if $\phi: \operatorname{MAX}\left(\ell_{n}^{1}\right) \rightarrow B(H)$ is any complete isometry, then the $C^{*}$-algebra $C^{*}\left(\left\{\phi\left(e_{i}\right)^{*} \phi\left(e_{j}\right): i, j=1, \ldots, n\right\}\right)$ has $C^{*}\left(\mathbb{F}_{n-1}\right)$ as a quotient via the map which sends $\phi\left(e_{i}\right)^{*} \phi\left(e_{j}\right) \rightarrow V_{i-1}^{*} V_{j-1} \in C^{*}\left(\mathbb{F}_{n-1}\right)$ where $V_{0}=I$.

In a similar fashion we can embed $\operatorname{MIN}\left(\ell_{n}^{1}\right)$ completely isometrically into $C\left(\mathbb{T}^{n-1}\right)$ by sending $e_{1}$ to the constant function 1 and $e_{i+1}$ to the $i$-th coordinate function $z_{i}$. Thus, $\operatorname{MAX}\left(\ell_{n}^{1}\right)$ generates the universal $C^{*}$-algebra for $n-1$ noncommuting unitaries while $\operatorname{MIN}\left(\ell_{n}^{1}\right)$ generates the universal $C^{*}$-algebra for $n-1$ commuting unitaries. 
Theorem 3.3. The number $\alpha\left(\ell_{n}^{1}\right)$ is the least positive constant $\alpha$ such that if $V_{1}, \ldots, V_{n-1}$ are any unitaries on a Hilbert space $H$, then there exist commuting unitaries $W_{1}, \ldots, W_{n-1}$ on a Hilbert space $K$ and operators $A \in B(K, H), B \in B(H, K)$ satisfying: $A B=I_{H}$, $V_{k}=A W_{k} B, k=1, \ldots, n-1$, and $\|A\|\|B\| \leqq \alpha$.

Proof. Regarding $\operatorname{MAX}\left(\ell_{n}^{1}\right)$ as a subspace of $C^{*}\left(\mathbb{F}_{n-1}\right)$ as above we have that there is a *-homomorphism sending $e_{i}$ to $V_{i-1}$, with $V_{0}=I_{H}$. Since the $c b$-norm of the identity map from $\operatorname{MIN}\left(\ell_{n}^{1}\right)$ to $\operatorname{MAX}\left(\ell_{n}^{1}\right)$ is $\alpha\left(\ell_{n}^{1}\right)$, we see that the map from $\operatorname{MIN}\left(\ell_{n}^{1}\right)$ into $B(H)$ which sends $e_{i}$ to $V_{i-1}$ has $c b$-norm at most $\alpha\left(\ell_{n}^{1}\right)$. Regarding $\operatorname{MIN}\left(\ell_{n}^{1}\right)$ as above as a subspace of $C\left(\mathbb{T}^{n-1}\right)$ and applying the generalized Stinespring theorem [9, Theorem 7.4] yields a unital *-homomorphism $\pi: C\left(\mathbb{T}^{n-1}\right) \rightarrow B(K)$, operators $A$ and $B$ as above such that $V_{i}=A \pi\left(z_{i}\right) B, I_{H}=A \pi(1) B=A B$, with $\|A\|\|B\|$ equal to the $c b$-norm of the map which is at most $\alpha\left(\ell_{n}^{1}\right)$.

To see that $\alpha\left(\ell_{n}^{1}\right)$ is sharp, let $V_{1}, \ldots, V_{n-1}$ be the generators of $C^{*}\left(\mathbb{F}_{n-1}\right)$.

Corollary 3.4. We have that $\alpha\left(\ell_{n}^{\infty}\right)=\alpha\left(\ell_{n}^{1}\right) \leqq \sqrt{n-1}$.

Proof. Let $V_{1}, \ldots, V_{n-1}$ be unitary operators on the Hilbert space $H$ and let $K=H^{(n-1)}$. Define $B: H \rightarrow K \quad$ via $B h=h \oplus V_{2} h \oplus \cdots \oplus V_{n-1} h \quad$ and $A: K \rightarrow H \quad$ via $A\left(h_{1} \oplus \cdots \oplus h_{n-1}\right)=h_{1}$ so that $A B=I_{H},\|A\|=1,\|B\|=\sqrt{n-1}$.

Let $S: K \rightarrow K$ denote the cyclic backwards shift $S\left(h_{1} \oplus \cdots \oplus h_{n-1}\right)=h_{2} \oplus \cdots \oplus h_{n-1} \oplus h_{1}$ and define commuting unitaries $W_{1}, \ldots, W_{n-1}$ on $K$ by $W_{1}\left(h_{1} \oplus \cdots h_{n-1}\right)=V_{1} h_{1} \oplus \cdots \oplus$ $V_{1} h_{n-1}$, and $W_{k}=S^{k-1}, k=2, \ldots, n-1$. Then $A W_{k} B=V_{k}$ as desired and so by Theorem $3.3, \alpha\left(\ell_{n}^{\prime}\right) \leqq \sqrt{n-1}$.

Combining this estimate with those obtained in [10] we have that

$$
\sqrt{n / 2} \leqq \alpha\left(\ell_{n}^{1}\right) \leqq \sqrt{n-1} \text { for } n \geqq 2 \text {. }
$$

\section{The space $C B(\operatorname{MIN}(X), \operatorname{MAX}(Y))$}

Let $X$ and $Y$ be any Banach spaces, since $\mathrm{MIN}(X)$ is the smallest operator space structure on $X$ and $\operatorname{MAX}(Y)$ is the largest operator space structure on $Y$ any map which is completely bounded from $\operatorname{MIN}(X)$ to $\operatorname{MAX}(Y)$ will automatically be completely bounded from $X$ to $Y$ for any other operator space structures on $X$ and $Y$. That is, $C B(\operatorname{MIN}(X), \operatorname{MAX}(Y))$ is the set of maps from $X$ to $Y$ which are completely bounded independent of the particular operator space structures that $X$ and $Y$ are assigned. In this section we prove that $C B(\operatorname{MIN}(X), \operatorname{MAX}(Y))=\Gamma_{2}^{*}(X, Y)$ and then apply this result to obtain some equivalences of tensor product norms which are analogous to Grothendieck's results. Recall $[11, \mathrm{p}$. 28] that $T: X \rightarrow Y$ belongs to $\Gamma_{2}^{*}(X, Y)$ if there exists a Hilbert space $H$ and maps $A: X \rightarrow H, B: H \rightarrow Y$ with $A$ and $B^{*}$ 2-summing and $T=B A$. For $T$ in $\Gamma_{2}^{*}(X, Y)$ we set $\gamma_{2}^{*}(T)=\inf \left\{\pi_{2}(A) \pi_{2}\left(B^{*}\right): T=B A\right\}$ 
where $\pi_{2}(\cdot)$ denotes the 2-summing norm and the infimum is taken over all factorizations of $T$ through a Hilbert space.

We define a universal constant via

$$
k=\sup \left\{n / \alpha\left(\ell_{n}^{2}\right): n=1,2, \ldots\right\} .
$$

By [10, Theorem 2.16], we have $\sqrt{2} \leqq k \leqq 2$. It is not hard to show that

$$
\alpha\left(\ell_{n+1}^{2}\right) \leqq \frac{n+1}{n} \alpha\left(\ell_{n}^{2}\right)
$$

and so the sequence of numbers $k_{n}=n / \alpha\left(\ell_{n}^{2}\right)$ is non-decreasing with $k=\lim _{n \rightarrow \infty} k_{n}$. Moreover, $k_{2}=\sqrt{2}$ and for $n>2$ exact values of $k_{n}$ (respectively $\alpha\left(\ell_{n}^{2}\right)$ ) are now known.

The goal of this section is to prove:

Theorem 4.1. Let $X$ and $Y$ be Banach spaces, then $C B(\operatorname{MIN}(X), \operatorname{MAX}(Y))=\Gamma_{2}^{*}(X, Y)$, with $\frac{1}{k} \gamma_{2}^{*}(T) \leqq\|T\|_{c b} \leqq \gamma_{2}^{*}(T)$. Moreover, the constant $k$ is the smallest constant that works for all Banach spaces.

Before beginning the proof we make a few remarks. First recall [11, Proposition 1.9] that for maps betweeen Hilbert spaces the 2-summing norm agrees with the HilbertSchmidt norm and hence the $\gamma_{2}^{*}$-norm is the trace norm.

Mimicking the proof of [8, Proposition 3.1] it readily follows that:

Lemma 4.2. Let $T \in C B\left(\operatorname{MIN}\left(\ell_{n}^{2}\right), \operatorname{MAX}\left(\ell_{n}^{2}\right)\right)$, then $k_{n}^{-1}\|T\|_{1} \leqq\|T\|_{c b} \leqq\|T\|_{1}$, where $\|\cdot\|_{1}$, is the trace norm.

Proof. Let $T=U D V$ be the singular value decomposition of $T$ with $U, V$ unitary and $D$ a positive diagonal matrix with diagonal entries, $d_{1}, \ldots, d_{n}$. Consider the commuting diagram,

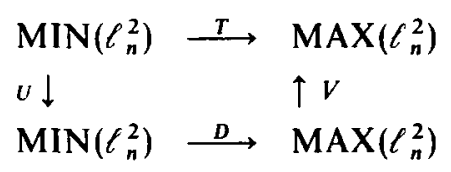

Since $\|U\|_{c b}=\|V\|_{c b}=\left\|U^{-1}\right\|_{c b}=\left\|V^{-1}\right\|_{c b}=1$, we have that $\|T\|_{c b}=\|D\|_{c b}$ and also $\|T\|_{1}=$ $\|D\|_{1}=\operatorname{tr}(D)$. Let $S$ denote the cyclic shift of order $n$ and note that $D+S D S^{-1}+\cdots+$ $S^{n-1} D S^{1-n}=\operatorname{tr}(D) I_{n}$. Hence, $\alpha\left(\ell_{n}^{2}\right) \cdot \operatorname{tr}(D)=\left\|\operatorname{tr}(D) I_{n}\right\|_{c b} \leqq\|D\|_{c b}+\cdot+\left\|S^{n-1} D S^{1-n}\right\|_{c b}=n\|D\|_{c b}$ and we have $k_{n}^{-1} \operatorname{tr}(D) \leqq\|D\|_{c b}$. On the other hand $D=d_{1} E_{11}+\cdots+d_{n} E_{n n}$ and hence $\|D\|_{c b} \leqq d_{1}\left\|E_{11}\right\|_{c b}+\cdots+d_{n}\left\|E_{n n}\right\|_{c b}=\operatorname{tr}(D)$ since $\left\|E_{i i}\right\|_{c b}=1$.

Considering the identity map shows that the above inequality is sharp. Note that the above result proves Theorem 4.1 for the special case where $X$ and $Y$ are finite dimensional Hilbert spaces, since as remarked earlier $\|T\|_{1}=\gamma_{2}^{*}(T)$ in that case. Moreover it shows that $k$ is the smallest constant which satisfies the above inequality for all finite dimensional Hilbert spaces. 
Also, we remark that it follows directly from the definitions that for a map $A: X \rightarrow H$ into a Hilbert space, the 2-summing norm $\pi_{2}(A)$ and the completely bounded norm of $A$ viewed as a map from $\operatorname{MIN}(X)$ to either $H_{\text {row }}$ or $H_{\text {column }}$ all coincide (see also [5]). Thus, using the operator space dualities, $\left(H_{\text {row }}\right)^{*}=H_{\text {cotumn }}, \operatorname{MAX}(Y)^{*}=\operatorname{MIN}\left(Y^{*}\right)$ we have also that for a map $B: H \rightarrow Y, \pi_{2}\left(B^{*}\right)$ and the completely bounded norms of $B$ viewed as a map from $H_{\text {row }}$ or $H_{\text {column }}$ into $\operatorname{MAX}(Y)$ all coincide.

Finally, if $E$ and $F$ are any operator spaces with $\operatorname{dim}(E)=n$ then $C B(E, F)=$ $B(E, F)=F \oplus \cdots \oplus F$ ( $n$ copies) and $C B(E, F)^{*}=F^{*} \oplus \cdots \oplus F^{*}=B(F, E)$ coincide as sets Following [6] we define the $O S$-integral norm on $B(F, E)$ via $\|R\|_{(F, E)}=\sup \{|\operatorname{tr}(R T)|$ : $\left.\|T\|_{C B(E, F)} \leqq 1\right\}$. It then follows trivially that $C B(E, F)^{*}$ can be identified with $B(F, E)$ endowed with the integral norm.

Lemma 4.3. Let $\mathrm{I}_{n}: \operatorname{MAX}\left(\ell_{n}^{2}\right) \rightarrow \operatorname{MIN}\left(\ell_{n}^{2}\right)$ denote the identity map, then

$$
\left\|I_{n}\right\|_{I\left(\operatorname{MAX}\left(\ell_{n}^{2}\right), \operatorname{MIN}\left(\ell_{n}^{2}\right)\right)}=k_{n} .
$$

Proof. If $T: \operatorname{MIN}\left(f_{n}^{2}\right) \rightarrow \operatorname{MAX}\left(\ell_{n}^{2}\right)$ then by Lemma $4.2,|\operatorname{tr}(T)| \leqq\|T\|_{1} \leqq k_{n}\|T\|_{c b}$ and so $\left\|I_{n}\right\|_{1} \leqq k_{n}$. To see equality, let $T=\alpha\left(\ell_{n}^{2}\right)^{-1} I_{n}$.

Lemma 4.4. Let $E_{1}, E_{2}, F_{1}$ and $F_{2}$ be operator spaces with $E_{1}$ and $E_{2}$ finite dimensional and assume

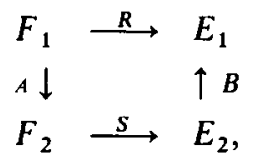

commutes.

Then $\|R\|_{1} \leqq\|A\|_{c h}\|S\|_{I}\|B\|_{c b}$

Proof. Note that

$$
\begin{aligned}
\|R\|_{I} & =\sup \left\{|\operatorname{tr}(B S A T)|\|T\|_{C B\left(E_{1}, F_{1}\right)} \leqq 1\right\} \\
& =\sup \left\{|\operatorname{tr}(S A T B)|:\|T\|_{C B\left(E_{1}, F_{1}\right)} \leqq 1\right\} \\
& \leqq \sup \left\{|\operatorname{tr}(S V)|:\|V\|_{C B\left(E_{2}, F_{2}\right)} \leqq\|A\|_{C b}\|B\|_{C b}\right\} \\
& =\|S\| I\|A\|_{c b}\|B\|_{C b}
\end{aligned}
$$

Proof of Theorem 4.1. Let $T \in \Gamma_{2}^{*}(X, Y)$ and write $T=B A$ with $B$ and $A$ 2-summing $A: X \rightarrow H, B: H \rightarrow Y$. Regarding $A$ and $B$ as maps from $\operatorname{MIN}(X)$ to $H_{\text {row }}$ and to $\operatorname{MAX}(Y)$, respectively, we see that $T \in C B(\operatorname{MIN}(X), \quad \operatorname{MAX}(Y))$ and $\|T\|_{c b} \leqq\|A\|_{c b}\|B\|_{c b}=$ $\pi_{2}(A) \pi_{2}\left(B^{*}\right)$. Thus, $\Gamma_{2}^{*}(X, Y) \subseteq C B(\operatorname{MIN}(X), \operatorname{MAX}(Y))$ with $\|T\|_{c b} \leqq \gamma_{2}^{*}(T)$.

To prove the other containment and inequality we first assume that $X$ is finite dimensional. From [11, Corollary 2.9 and the Remark on p. 28] we know that $\left(\Gamma_{2}^{*}(X, Y)\right)^{*}=\Gamma_{2}(Y, X)$ where for $R: Y \rightarrow X, \gamma_{2}(R)=\inf \{\|A\|\|B\|\}$ and the infimum is taken over all factorizations of $R$ through Hilbert space. By the above remarks we know that $C B(\operatorname{MIN}(X), \operatorname{MAX}(Y))^{*}=B(Y, X)$ with the $O S$-integral norm. Thus proving that 


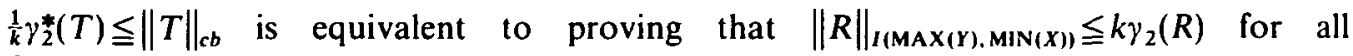
$R \in B(Y, X)$.

Let $R=A B$ where $B: Y \rightarrow \ell_{n}^{2}, A: \ell_{n}^{2} \rightarrow X$ we have

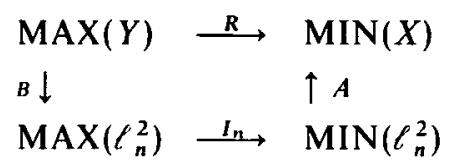

and hence $\|R\|_{I} \leqq\|A\|_{c b}\left\|I_{n}\right\|_{I}\|B\|_{c b} \leqq k\|A\|\|B\|$ from which $\|R\|_{I} \leqq k \gamma_{2}(R)$ follows.

Now to prove the inequality for a general $X$ let $F \subseteq X$ be a finite dimensional subspace and let $T_{F}$ denote the restriction of $T$ to $F$. Since $\operatorname{MIN}(F) \subseteq \operatorname{MIN}(X)$ completely isometrically, it follows that a map $T: X \rightarrow Y$ is completely bounded from $\operatorname{MIN}(X)$ to $\operatorname{MAX}(Y)$ if and only if $\sup \left\{\left\|T_{F}\right\|_{C B(\operatorname{MIN}(F), \operatorname{Max}(Y))}: \operatorname{dim}(F)<+\infty\right\}$ is finite and that this supremum is $\|T\|_{C B(\operatorname{MIN}(X), \operatorname{MAX}(Y))}$. Thus, $\|T\|_{C B(\operatorname{MIN}(X), \operatorname{MAX}(Y))} \geqq \frac{1}{k} \sup \left\{\gamma_{2}^{*}\left(T_{F}\right)\right.$ : $\operatorname{dim}(F)<+\infty\}$.

It remains to show that if $T: X \rightarrow Y$ satisfies $\gamma_{2}^{*}\left(T_{F}\right)<C$ for every finite dimensional $F \subseteq X$, then necessarily $T \in \Gamma_{2}^{*}(X, Y)$ and $\gamma_{2}^{*}(T) \leqq C$. This follows from arguments similar to those given in the proof of [11, Proposition 2.3] and is certainly known. However, for the sake of completeness and to illustrate some of the interplay between $c b$-maps and these purely Banach space concepts we give an alternate proof of this fact, using $c b$-techniques.

To this end let $R_{x}=E_{11} \cdot B\left(\ell^{2}\right)$ denote an infinite dimensional row space. For each finite dimensional $F \subseteq X$ we have a factorization

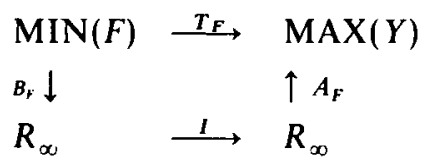

such that $\left\|B_{F}\right\|_{c b}=\pi_{2}\left(B_{F}\right)=1,\left\|A_{F}\right\|_{c b}=\pi_{2}\left(A_{F}^{*}\right)<C$. Since $\operatorname{MIN}(F) \subseteq \operatorname{MIN}(X)$, completely isometrically, and $R_{\infty}$ is an injective operator space, there exists $B_{F}^{\prime}: \operatorname{MIN}(X) \rightarrow R_{\infty}$ which extends $B_{F}$ and has $\left\|B_{F}^{\prime}\right\|_{c b}=\left\|B_{F}\right\|_{c b}=1$. Since the unit ball of $C B\left(\operatorname{MIN}(X), R_{\infty}\right)$ is compact in the $B W$-topology (see [9, Chapter 5]) there exists $B: \operatorname{MIN}(X) \rightarrow R_{\infty},\|B\|_{c b} \leqq 1$ which is the limit of some subnet $\left\{B_{F}: \lambda \in \Lambda\right\}$ of the net $\left\{B_{F}^{\prime}: F \in \mathbb{F}\right\}$ where $\mathfrak{F}$ denotes the directed set of finite dimensional subspaces.

The closed subspace $R=B(X)^{-}$of $R_{\infty}$ is easily seen to be a Hilbert row operator space. Define $A: B(X) \rightarrow \operatorname{MAX}(Y)$ via $A(B x)=T x$. Given $x_{i j} \in X, i, j=1, \ldots, n$ let $F=$ $\operatorname{span}\left\{x_{i j}: i, j=1, \ldots, n\right\}$ then there exists $\lambda_{0}$ such that for $\lambda_{0}, F \subseteq F_{i}$ and hence, $\left\|\left(A\left(B x_{i j}\right)\right)\right\|_{M_{n}(\operatorname{MAXX}(Y))}=\left\|\left(T x_{i j}\right)\right\|_{M_{n}(\operatorname{MAX}(Y))}=\left\|\left(A_{F ;}\left(B_{F} ; x_{i j}\right)\right)\right\|_{M_{n}(\operatorname{MAX}(Y))} \leqq C\left\|\left(B_{F} ;\left(x_{i j}\right)\right)\right\|_{M_{n}\left(R_{o w}\right)}$ for all $\lambda \geqq \lambda_{0}$. Hence, $\left\|\left(A\left(B x_{i j}\right)\right)\right\| \leqq C\left\|\left(B\left(x_{i j}\right)\right)\right\|$, and so $A$ extends to a completely bounded map on $R$ with $C \geqq\|A\|_{c b}=\pi_{2}\left(A^{*}\right)$. Thus, we have that $T=A B$ is a factorization of $T$ with $\pi_{2}(B) \pi_{2}\left(A^{*}\right) \leqq C$. This completes the proof of the theorem.

In the following $\otimes_{h}, \widehat{\otimes}$, and $\otimes$ denote the Haagerup, operator space projective, and 
spatial tensor products, respectively (see [3] for definitions, unfortunately with different notation).

Corollary 4.5. Let $X$ and $Y$ be any Banach spaces and let $u \in X \otimes Y$, then

$$
\begin{aligned}
\|u\|_{M I N(X) \otimes_{h} \operatorname{MIN}(Y)} & \leqq u \|_{M I N(X) \bar{\otimes} \operatorname{MIN}(Y)} \\
& \leqq k\|u\|_{\operatorname{MIN}(X) \otimes_{n} \operatorname{MIN}(Y)}
\end{aligned}
$$

and

$$
\begin{aligned}
\|u\|_{\operatorname{MAX}(Y) \otimes \operatorname{MAX}(Y)} & \leqq u \|_{\operatorname{MAX}(X) \otimes_{h \operatorname{MAX}(Y)}} \\
& \leqq k\|u\|_{\operatorname{MAX}(X) \otimes \operatorname{MAX}(Y)}
\end{aligned}
$$

Proof. Given $u \in X \otimes Y$ let $T_{u}: \operatorname{MAX}(X)^{*} \rightarrow \operatorname{MAX}(Y)$ be the associated finite rank operator and recall $\operatorname{MAX}(X)^{*}=\operatorname{MIN}\left(X^{*}\right)$.

By $\left[3\right.$, Corollary 5.2], $\left\|T_{u}\right\|_{C B\left(\operatorname{MIN}\left(X^{*}\right), \operatorname{MAX}(Y)\right)}=\|u\|_{\operatorname{MAX}(X) \otimes \operatorname{MaX}(Y)} \leqq\|u\|_{\operatorname{MAX}(X) \otimes_{h} \operatorname{MAX}(Y)}$. While by Theorem 4.1, $\left\|T_{u}\right\|_{C B\left(\operatorname{MIN}\left(X^{*}\right), \operatorname{MAX}(Y)\right)} \geqq \frac{1}{k} \gamma_{2}^{*}\left(T_{u}\right)=\frac{1}{k}\|u\|_{\operatorname{MAX}(X) \otimes_{h} \operatorname{MAX}(Y)}$ by $\quad[1$, Theorem 3.1].

The other set of inequalities comes for $X$ and $Y$ finite dimensional by dualizing the above set and using the facts that the dual norm of the spatial is the operator space projective for finite dimensional spaces by [3, Theorem 5.6] while the Haagerup norm is self-dual [5]. To pass to $X$ and $Y$ infinite dimensional one use that $\operatorname{MIN}(\cdot)$, and the Haagerup tensor behave injectively while the projective tensor behaves projectively.

We are grateful to D. Blecher for pointing out the following fact.

Corollary 4.6. Let $H$ and $K$ be Hilbert-spaces then

$$
\begin{aligned}
\|u\|_{\mathrm{MIN}(H) \otimes \mathrm{MIN}(K)} & \leqq u \|_{\mathrm{MIN}(H) \bar{\otimes} \operatorname{MIN}(K)} \\
& \leqq k\|u\|_{\mathrm{MIN}(H) \otimes \operatorname{MIN}(K)}
\end{aligned}
$$

and

$$
\begin{aligned}
\|u\|_{\operatorname{MAX}(H) \otimes \operatorname{MAX}(K)} & \leqq u \|_{\operatorname{MAX}(H) \otimes \operatorname{MAX}(K)} \\
& \leqq k\|u\|_{\operatorname{MAX}(H) \otimes \operatorname{MAX}(K)} .
\end{aligned}
$$

Consequently, all operator space tensor norms on these spaces are equivalent.

Proof. The first inequalities come from observing that $\|u\|_{\operatorname{MIN}(H) \otimes \operatorname{Min}(K)}=$ $\|u\|_{\operatorname{MIN}(H) \otimes_{n} \operatorname{MIN}(K)}$. To see this recall that if $T_{u}: \operatorname{MIN}(H)^{*} \rightarrow \operatorname{MIN}(K)$ then by $[3$, Theorem 11], $\quad\|u\|_{\operatorname{MIN}(H) \otimes_{n} \operatorname{MIN}(K)}=\inf \left\{\|A\|_{c b}\|B\|_{c b}: T_{u}=A B, B: \operatorname{MIN}(H)^{*} \rightarrow H_{R}, A: R_{n} \rightarrow \operatorname{MIN}(K)\right\}$ where $H_{R}$ denotes a row Hilbert space. We may let $B$ be the identity map and $A=T_{u}$. Then since $\operatorname{MIN}(H)^{*}=\operatorname{MAX}(H),\|B\|_{c b}=\|B\|=1$ while since $\operatorname{MIN}(K)$ is a min space, $\|A\|_{c b}=\|A\|=\left\|T_{u}\right\|_{C B\left(\operatorname{MIN}(H)^{*} \cdot \operatorname{MIN}(K)\right)}=\|u\|_{\operatorname{MiN}(H) \Theta \operatorname{MIN}(K)}$. 
Hence,

$$
\|u\|_{\operatorname{MIN}(H) \otimes h \operatorname{MIN}(K)} \leqq\|u\|_{\operatorname{MIN}(H) \otimes \operatorname{MIN}(K)}
$$

from which equality follows.

The remaining inequalities come by duality as in Corollary 4.5 .

1 do not know if the inequalities in Corollary 4.5 or 4.6 are true at the matrix level.

Remark 4.6. Recall that Grothendieck's inequality says that when $X$ and $Y$ are $\ell^{\infty}$-spaces, then $\|u\|_{X \otimes_{H} Y} \leqq u \|_{X \otimes Y}$, where $\hat{\otimes}$ and $\otimes_{H}$ denotes the projective Banach space and Hilbertian tensor products, respectively. Since by [1] $X \otimes_{H} Y=\operatorname{MIN}(X)$ $\otimes_{h} \operatorname{MIN}(Y)$, isometrically for all spaces $X$ and $Y$, Corollary 4.5 can be seen as an analogue of Grothendieck's inequality where the Banach space projective tensor norm has been replaced by the smaller operator space projective norm. However at the same time we've gained since in Corollary $4.5 X$ and $Y$ no longer neeed be $\ell^{\infty}$-spaces.

Grothendieck's inequality implies that when $X$ and $Y$ are $\ell^{\infty}$-spaces $X \hat{\otimes} Y$ and $\operatorname{MIN}(X) \widehat{\otimes} \operatorname{MIN}(Y)$ have equivalent norms. Conversely, this fact together with Corollary 4.5 imply Grothendieck's inequality. Thus an independent proof of this last equivalence of norms would yield a new proof of Grothendieck's inequality. It is unclear which of these two inequalities is most closely related to Grothendieck's inequality, or whether they parse it.

To put this more clearly, identify a typical element $u=\sum a_{i j} e_{i} \otimes e_{j}$ of $\ell_{n}^{\infty} \otimes \ell_{m}^{\infty}$ with the $n \times m$ matrix $\left(a_{i, j}\right)$. If we let $B_{1}, B_{2}$ and $B_{3}$ denote the unit balls in $\ell_{n}^{\infty} \otimes \ell_{n}^{\infty}$, $\operatorname{MIN}\left(\ell_{n}^{\infty}\right) \hat{\otimes} \operatorname{MIN}\left(\ell_{m}^{\infty}\right)$ and $\operatorname{MIN}\left(\ell_{n}^{\infty}\right) \otimes_{h} \operatorname{MIN}\left(\ell_{m}^{\infty}\right)=\ell_{n}^{\infty} \otimes_{H} \ell_{m}^{\infty}$, respectively, then it can be shown that

$$
\begin{aligned}
& B_{1}=\operatorname{convh}\left\{\left(\alpha_{i} \beta_{j}\right):\left|\alpha_{i}\right| \leqq 1,\left|\beta_{j}\right| \leqq 1\right\} \\
& B_{2}=\left\{\left(\left\langle A_{i} \otimes C_{j} x, y\right\rangle\right):\left\|A_{i}\right\| \leqq 1,\left\|C_{j}\right\| \leqq 1,\|x\| \leqq 1,\|y\| \leqq 1\right\} \\
& B_{3}=\left\{\left(\left\langle x_{i}, y_{j}\right\rangle\right):\left\|x_{i}\right\| \leqq 1,\left\|y_{j}\right\| \leqq 1\right\}
\end{aligned}
$$

where the vectors $x_{i}, y_{j}$ are vectors in some Hilbert space, the operators $A_{i}, C_{j}$ are operators on Hilbert spaces $H$ and $K$, respectively, with $x, y \in H \otimes K$. We have that $B_{1} \subseteq B_{2} \subseteq B_{3}$, Grothendieck's inequality implies that $B_{3} \subseteq K_{\mathrm{C}} \cdot B_{1}$ where $K_{\mathrm{C}}$ is the complex Grothendieck constant, while Corollary 4.5 implies that $B_{3} \subseteq k \cdot B_{2}$. Hence, we know that there exists a constant $c$ such that $B_{2} \subseteq c B_{1}$ independent of $n$ and $m$. Our question is whether or not the existence of such a constant $c$ is less deep than the original Grothendieck inequality.

Corollary 4.7. Let $U_{1}, \ldots, U_{n}, V_{1}, \ldots, V_{m}$ be unitaries on the Hilbert space $H$ which generate $C^{*}\left(\mathbb{F}_{m+n}\right)$, then for any scalars $\alpha_{i j}$,

$$
\left\|\sum \alpha_{i j} U_{i} V_{j}\right\|_{B(H)} \leqq k\left\|\sum \alpha_{i j} U_{i} \otimes V_{j}\right\|_{B(H \otimes H)} .
$$


Proof. Recall that $\operatorname{MAX}\left(\ell_{n}^{1}\right)=\operatorname{span}\left\{U_{1}, \ldots, U_{n}\right\}, \operatorname{MAX}\left(\ell_{m}^{1}\right)=\operatorname{span}\left\{V_{1}, \ldots, V_{m}\right\}$ completely isometrically. By [4, Theorem 3.1], and the injectivity of the Haagerup tensor norm, $\operatorname{MAX}\left(\ell_{n}^{1}\right) \otimes_{h} \operatorname{MAX}\left(\ell_{m}^{1}\right)=\operatorname{span}\left\{U_{i} * V_{j}\right\} \subseteq C^{*}\left(\mathbb{F}_{n}\right) * C^{*}\left(\mathbb{F}_{m}\right)$. Since $C^{*}\left(\mathbb{F}_{n+m}\right)$ is the quotient of $C^{*}\left(\mathbb{F}_{n}\right) * C^{*}\left(\mathbb{F}_{m}\right)$ obtained by amalgamating along the identity, the result follows from Corollary 4.5 .

Remark 4.8. Let $T: \ell_{1}^{n} \rightarrow \ell_{1}^{n}$, say $T=\left(t_{i j}\right)$ and assume $T$ is factored as $T=A B$, $B: \ell_{1}^{n} \rightarrow \ell_{2}^{m}, A: \ell_{2}^{m} \rightarrow \ell_{1}^{n}$. The identification of $\mathrm{MIN}\left(\ell_{1}^{n}\right)$ with the span of $n$ commuting unitaries and the inequality $\|T\|_{c b} \leqq \gamma_{2}^{*}(T)$ implies that for any $n$ unitaries $\left\{U_{1}, \ldots, U_{n}\right\}$ there exists a dilation of the following form:

$$
\sum_{i=1}^{n} t_{i j} U_{i}=X Z_{j} Y, \quad j=1, \ldots, n
$$

where $\left\{Z_{1}, \ldots, Z_{n}\right\}$ are commuting unitaries and $\|X\|\|Y\| \leqq \pi_{2}(B) \pi_{2}\left(A^{*}\right)$.

It seems likely that one should be able to use the matrices $A$ and $B$ to construct such a dilation explicitly but we have been unable to see how to do this.

We close this paper with a final formula for the constants $k_{m}$.

Theorem 4.9. Let $k_{n}=n / \alpha\left(\ell_{n}^{2}\right)$ then

$$
k_{n}=\left\|\sum_{i=1}^{n} e_{i} \otimes e_{i}\right\|_{\operatorname{MIN}\left(\ell_{n}^{2}\right) \otimes \operatorname{MIN}\left(\ell_{n}^{2}\right)}=\inf \left\{\|x\|\|y\|: \delta_{i j}=\left\langle\left(A_{i} \otimes B_{j}\right) x, y\right\rangle\right\}
$$

where $A_{1}, \ldots, A_{n} \in B(H), B_{1}, \ldots, B_{n} \in B(K)$ with $H$ and $K$ Hilbert spaces are operators satisfying

$$
\left\|\sum \lambda_{i} A_{i}\right\| \leqq 1,\left\|\sum \lambda_{i} B_{i}\right\| \leqq 1 \text { for all } \sum\left|\lambda_{i}\right|^{2} \leqq 1
$$

and $x, y \in H \otimes K$.

Proof. Recall $I\left(\operatorname{MAX}\left(\ell_{n}^{2}\right), \operatorname{MIN}\left(\ell_{n}^{2}\right)\right)=C B\left(\operatorname{MIN}\left(\ell_{n}^{2}\right), \operatorname{MAX}\left(\ell_{n}^{2}\right)\right)^{*}$ and by [3] the identification of $u \in \operatorname{MAX}\left(\ell_{n}^{2}\right) \otimes \operatorname{MAX}\left(\ell_{n}^{2}\right)$ with $T_{u} \in C B\left(\operatorname{MIN}\left(\ell_{n}^{2}\right), \operatorname{MAX}\left(\ell_{n}^{2}\right)\right)$ is an isometry. When $T_{u}=I$, then $u=\sum_{i=1}^{n} e_{i} \otimes e_{i}$, Hence by Lemma 4.3 and [3],

$$
\begin{aligned}
k_{n}=\left\|I_{n}\right\|_{C B\left(\operatorname{MiN}\left(\ell_{n}^{2}\right), \operatorname{MAX}\left(\ell_{n}^{2}\right)\right)^{*}} & =\left\|\sum_{i=1}^{n} e_{i} \otimes e_{i}\right\|_{\left(\operatorname{MAX}\left(\ell_{n}^{2}\right) \otimes \operatorname{MAX}\left(\ell_{n}^{2}\right)\right)^{*}} \\
& =\left\|\sum_{i}^{n} e_{i} \otimes e_{i}\right\|_{\operatorname{MIN}\left(\ell_{n}^{2}\right) \otimes \operatorname{MIN}\left(\ell_{n}^{2}\right) .}
\end{aligned}
$$

Now by the characterization in [6] of the projective operator cross-norm, we have that 


$$
\|u\|_{\operatorname{MIN}\left(\ell_{n}^{2}\right) \otimes \operatorname{MIN}\left(\ell_{n}^{2}\right)}=\inf \{\|y\|\|x\|: u=y(A \otimes B) x\}
$$

where $y$ is a row vector, $x$ is a column vector, $A \in M_{r}\left(\operatorname{MIN}\left(\ell_{n}^{2}\right)\right) B \in M_{t}\left(\operatorname{MIN}\left(\ell_{n}^{2}\right)\right)$ with $\|A\| \leqq 1,\|B\| \leqq 1$. Using the canonical basis we may write

$$
A=\sum A_{i} \otimes e_{i}, \quad B=\sum B_{j} \otimes e_{j}
$$

where each $A_{i}$ is a scalar $r \times r$ matrix and each $B_{j}$ is a scalar $t \times t$ matrix. When $u=\sum_{i=1}^{n} e_{i} \otimes e_{i}$ we have that $y\left(A_{i} \otimes B_{j}\right) x=\delta_{i j}$.

\section{REFERENCES}

1. D. B. Blecher, Tensor products of operator spaces II, Canad. J. Math. 44 (1992), 75-90.

2. D. B. Blecher, The standard dual of an operator space, Pacific J. Math. 153 (1992), 15-30.

3. D. B. Blecher and V. I. Paulsen, Tensor products of operator spaces, J. Funct. Anal. 99 (1991), 262-292.

4. E. Christensen, E. Effros and A. Sinclair, Completely bounded maps and $C^{*}$ algebraic cohomology, Invent. Math. 90 (1987), 279-296.

5. E. Effros and Z. J. RuAN, Self duality for the Haagerup tensor product and Hilbert space factorization, J. Funct. Anal. 100 (1991), 257-284.

6. E. Effros and Z. J. RuAN, The Grothendieck-Pietsch and Dvoretsky-Rogers theorems for operator spaces, J. Funct. Anal., to appear.

7. M. JUnge and G. Pisier, Bilinear forms on exact operator spaces and $B(H) \otimes B(H)$, GAFA 5 (1995), 329-363.

8. D. B. Mathes and V. I. Paulsen, Operator ideals and operator spaces, Proc. Amer. Math. Soc. 123 (1995), 1763-1772.

9. V. I. Paulsen, Completely bounded maps and dilations (Pitman Research Notes, 146, Longman, 1986).

10. V. I. PAulsEn, Representations of function algebras, abstract spaces and Banach space geometry, J. Funct. Anal. 109 (1992), 113-129.

11. G. PISIER, Factorization of linear operators and the geometry of Banach spaces (CBMS Regional Conference Series 60, 1986), reprinted 1987.

12. Z. J. Ruan, Subspaces of $C^{*}$-algebras, J. Funct. Anal. 76 (1988), 217-230.

13. C. Zhang, Representation and Geometry of Operator Spaces (Thesis, University of Houston, 1994).

14. C. Zhang, Representations of operator spaces, J. Operator Th. 33 (1995), 327-351.

Department of Mathematics

UNIVERITY OF HOUSTON

TEXAS 77204-3476

U.S.A. 